\title{
Hepatic HNF1 transcription factors control the induction of PCSK9 mediated by rosuvastatin in normolipidemic hamsters
}

\author{
BIN DONG, AMAR BAHADUR SINGH, VIKRAM RAVINDRA SHENDE and JINGWEN LIU \\ Department of Veterans Affairs, Palo Alto Health Care System, Palo Alto, CA 94304, USA
}

Received May 24, 2016; Accepted January 26, 2017

DOI: 10.3892/ijmm.2017.2879

\begin{abstract}
Proprotein convertase subtilisin/kexin type 9 (PCSK9) impedes low-density lipoprotein (LDL) receptor (LDLR)-mediated LDL-cholesterol uptake and has hence emerged as a critical regulator of serum cholesterol levels and a new therapeutic target for the treatment of hypercholesterolemia. Statins have been shown to elevate circulating PCSK9 levels by stimulating PCSK9 gene transcription, which reduces the clinical efficacy of statin in LDL-cholesterol reduction. The transcription of PCSK9 is partially controlled by the hepatocyte nuclear factor 1 (HNF1) binding site embedded in the proximal region of its promoter. In this study, we utilized adenoviral shRNA delivery vectors to generate liver-specific knockdown of HNF1 $\alpha$ (Ad-shHNF1 $\alpha$ ) or HNF1 $\beta$ (Ad-shHNF1 $\beta$ ) in hamsters to examine the impact of reduced hepatic expression of HNF1 transcription factors on statin-induced elevation of PCSK9 expression and serum cholesterol levels. We showed that the administration of rosuvastatin (RSV) to normolipidemic hamsters significantly augmented hepatic PCSK9 expression and serum PCSK9 levels. In addition, RSV treatment increased hepatic HNF1 $\alpha$ protein levels without a clear effect on HNF1 $\alpha$ mRNA expression. Injection of Ad-shHNF1 $\alpha$ or Ad-shHNF1 $\beta$ into hamsters both blunted RSV-induced elevation of PCSK9 serum concentration and hepatic mRNA and protein levels, which led to significant increases in liver LDLR protein abundance. Furthermore, hepatic depletion of HNF1 factors lowered circulating total cholesterol and non-high density lipoprotein cholesterol levels in RSV-treated hamsters. Our study demonstrates that both HNF1 $\alpha$ and HNF1 $\beta$ are positive regulators of hepatic PCSK9 transcription in hamster species and that transient, liver-specific knockdown of either HNF1 $\alpha$ or HNF1 $\beta$
\end{abstract}

Correspondence to: Dr Jingwen Liu, Department of Veterans Affairs, Palo Alto Health Care System, 3801 Miranda Avenue, Palo Alto, CA 94304, USA

E-mail: jingwen.liu@va.gov

Key words: proprotein convertase subtilisin/kexin type 9, low-density lipoprotein receptor, hepatocyte nuclear factor $1 \alpha$ and hepatocyte nuclear factor $1 \beta$, statin, hyperlipidemia, hamsters could antagonize the RSV-induced elevation of serum PCSK9 and reduce circulating cholesterol levels.

\section{Introduction}

Proprotein convertase subtilisin/kexin type 9 (PCSK9) is a liver-derived plasma protein that regulates plasma low-density lipoprotein-cholesterol (LDL-C) levels by diverting the cell surface LDL receptor (LDLR) of hepatocytes to lysosomes for degradation $(1,2)$. Thus, PCSK9 plasma levels directly influence the level of circulating LDL-C $(3,4)$. Recent clinical studies have shown that a reduction in circulating PCSK9 using neutralizing anti-PCSK9 antibodies can lower serum LDL-C levels in dyslipidemic and hypercholesterolemic patients (5), which provide strong validation to support the notion that lowering circulating PCSK9 levels to upregulate hepatic LDLR is beneficial for reducing the risk of cardiovascular disease in humans.

In liver tissue, PCSK9 synthesis is largely controlled at the gene transcriptional level by two transcription factor families, sterol regulatory element-binding proteins (SREBPs) $(6,7)$ and hepatocyte nuclear factor 1 (HNF1) (8). PCSK9 gene expression is positively regulated by SREBP through an SRE motif of the proximal promoter in response to depletion of intracellular levels of sterols $(9,10)$. Statins, a class of cholesterol lowering drugs, increase the PCSK9 expression level by activating the SREBP pathway and thus diminish their beneficial effects (11). Our laboratory has previously identified a highly conserved HNF1 binding site on the PCSK9 promoter region as another critical regulatory sequence motif of PCSK9 transcription (8). DNA binding studies conducted in HepG2 cells identified HNF1 $\alpha$ as the primary transactivator that binds to the HNF1 site of PCSK9 promoter. We have shown that siRNA-mediated knockdown of HNF1 $\alpha$ reduced PCSK9 protein levels in HepG2 cells. In vivo, an increased binding of HNF1 $\alpha$ to the PCSK9 promoter was detected in the liver of rosuvastatin (RSV)-treated hamsters, which was thought to contribute to the strong induction of PCSK9 gene expression by RSV in that animal model (12).

HNF1 $\alpha$ and HNF1 $\beta$, the two members of the HNF1 family, are homeobox proteins that form homodimers or heterodimers to bind HNF1 sites on the promoter of their target genes $(13,14)$. The importance of HNF1 $\alpha$ in PCSK9 expression has been clearly demonstrated in cell culture studies and in 
mice where adenovirus-mediated overexpression of HNF1 $\alpha$ led to increased PCSK9 and reduced liver LDLR protein (15). Relative to HNF1 $\alpha$, studies to address the role of HNF1 $\beta$ in PCSK9 transcription are scarce. One earlier study reported that while HNF1 $\beta$ is normally expressed at low levels in adult mouse hepatocytes, its expression is induced in $\mathrm{HNF} 1 \alpha^{-1-}$ livers (16). In vivo DNA binding experiments indicated that loss of HNF1 $\alpha$ triggers higher HNF1 $\beta$ binding at several HNF1 $\alpha$ target loci, including the PCSK9 promoter region, implicating that HNF1 $\beta$ could also modulate PCSK9 gene expression. Previously, utilizing mouse models, our laboratory attempted to examine the functional roles of HNF1 $\alpha$ and HNF1 $\beta$ in PCSK9 expression in liver tissue by applying adenovirus-mediated delivery of shRNAs targeted to the HNF1 family members in mice. We found that in mice adenovirus-mediated liver-specific knockdown of HNF1 $\alpha$ markedly decreased serum PCSK9 and hepatic PCSK9 expression whereas depletion of HNF1 $\beta$ by the same approach had no effects (17). Thus, our studies provided direct evidence to support the notion that in mouse species HNF1 $\alpha$ but not HNF1 $\beta$ regulates liver PCSK9 expression.

Evidence exists that the expression of endogenous PCSK9 and its response to pharmacological and nutritional factors vary among different species including mice, hamsters and non-human primates (18-20). Since RSV has been shown to strongly induce PCSK9 expression in hamsters by activating the SREBP pathway and by increasing HNF1 $\alpha$ protein levels (12), in this study, we thus examined the effects of transient knockdown of HNF1 isoforms HNF1 $\alpha$ and HNF1 $\beta$ individually in hamster livers on secreted PCSK9 and hepatic LDLR expression under statin treatment conditions. Our results for the first time demonstrated that in hamster species, both HNF1 $\alpha$ and HNF1 $\beta$ are functionally involved in hepatic PCSK 9 transcription and that transient, liver-specific knockdown of either HNF1 $\alpha$ or HNF1 $\beta$ could antagonize the statin-induced elevation of serum PCSK9 and lower circulating cholesterol levels.

\section{Materials and methods}

Cloning of hamster HNFla complete coding sequence. When this project was started, sequences for the golden Syrian hamster HNF1 $\alpha$ gene were not available. By applying a homology based primer selection approach we amplified the whole coding sequence of HNF1 $\alpha$ hamster orthologue from a cDNA pool generated from male hamster liver samples and cloned the PCR product into pCR2.1-Topo vector (Invitrogen Life Technologies, Carlsbad, CA, USA). After sequencing the entire coding sequence, we cloned the hamster HNF1 $\alpha$ coding region into pcDNA4.0-HisMax-TOPO vector (Invitrogen Life Technologies) and transfected the plasmid into 293 cells (American Type Culture Collection, Manassas, VA, USA) to validate the correct expression of hamster HNF1 $\alpha$ protein. Recently, the genomic sequence of a female golden Syrian hamster (Mesocricetus auratus) became available on NCBI. The NCBI reference sequence (HNF1 $\alpha$ transcript X2, XM_005078961.1) predicted the HNF1 $\alpha$ nucleotide coding sequence and protein sequence that are $100 \%$ identical to our hamster HNF1 $\alpha$ cDNA and protein sequences.

shRNA design and adenoviral vector productions. Hamster hepatic HNF1 $\alpha$ and HNF1 $\beta$ were transiently knocked down using adenoviral vectors expressing shRNA targeting to HNF1 $\alpha$ or HNF1 $\beta$. Briefly, a sequence spanning hamster HNF1 $\alpha$ mRNA coding sequence from 786 to 806 that is identical to human, mouse, and rat sequences was selected as the target for RNAi. For HNF1 $\beta$, we cloned a partial coding region of hamster HNF1 $\beta$ and sequenced. The sequence of the partial clone was identical to the corresponding sequence of the NCBI-predicted HNF1 $\beta$ transcript X1. Thus, a sequence spanning nucleotides $1464-1484$ of the hamster HNF1 $\beta$ mRNA transcript X1 was targeted for shRNA-mediated knockdown. This sequence is identical among all isoforms of the hamster, human and mouse HNF1 $\beta$ sequence. To construct Ad-shHNF1 $\alpha /$ Ad-shHNF1 $\beta$ adenoviral vectors, a U6 promoter-based shuttle vector (pSH-HNF1 $\alpha$ or pSH-HNF1 $\beta$ ) that expresses HNF1 $\alpha$ or HNF1 $\beta$ shRNA was generated using BLOCK-iT ${ }^{\text {TM }}$ U6 RNAi entry vector kit (Invitrogen Life Technologies) following the manufacturer's instructions as previously described (17). Ad-shLacZ encoding an shRNA for the LacZ gene was used as control in this study.

Animals and adenoviral injection. Male Syrian golden hamsters were purchased from Harlan Laboratories, Inc. (Indianapolis, IN, USA) at 8-10 weeks of age and weighed 100-110 g. Animals were housed 2 per cage in the VA Palo Alto Health Care System (VAPAHCS) Veterinary Medical Unit under a $12 \mathrm{~h}$ light and dark cycle (6:00 a.m.-6:00 p.m.). Hamsters were fed a regular rodent chow diet and water ad libitum. Hamsters had a 7-day acclimation period before the experiments. All experimental analyses were conducted in accordance with animal use protocols approved by the Institutional Animal Care and Use Committee at the VAPAHCS. Hamster body weight and food intake were recorded every two days throughout the duration of the experiment. Animals were administered with $5 \times 10^{11}$ viral particles under isoflurane anesthesia via the orbital venous plexus. Three days post injection, animals were orally administered with RSV at a daily dose of $15 \mathrm{mg} / \mathrm{kg}$ for seven days. The control group (Ad-shLacZ) received an equal volume of the vehicle (sterilized water).

Blood samples were collected by administering isoflurane and bleeding from the orbital venous plexus in lithium heparinized capillary tubes before the study and at the end of the study. At the termination, animals were dosed with RSV and the food was removed. Blood and liver were harvested after a 4-h fast. Livers were collected, weighed, frozen in liquid nitrogen, and stored at $-80^{\circ} \mathrm{C}$ until processing. RSV was purchased from AK Scientific, Inc. (Union City, CA, USA). Serum levels of total cholesterol and HDL cholesterol were measured using respective kits from Stanbio Laboratory, (Boerne, TX, USA) according to the manufacturer's instructions. Non-HDL-C was calculated as total cholesterol minus HDL-C. Serum ALT activity was measured using the ALT/SGPT Liqui-UV kit (Stanbio Laboratory) following the manufacturer's instructions.

Detection of hamster PCSK9 in serum. Levels of hamster serum PCSK9 were measured using the mouse PCSK9 quantikine ELISA kit (R\&D Systems, Inc., Minneapolis, MN, USA) (21). Briefly, serum samples were diluted 1:10 in calibrator diluent and allowed to bind for $2 \mathrm{~h}$ onto microplate wells that were precoated with the capture antibody. Samples were 
Table I. List of hamster primers for RT-qPCR.

\begin{tabular}{lll}
\hline Gene & \multicolumn{1}{c}{ Forward $\left(5^{\prime} \rightarrow 3^{\prime}\right)$} & \multicolumn{1}{c}{ Reverse $\left(5^{\prime} \rightarrow 3^{\prime}\right)$} \\
\hline GAPDH & AACTTTGGCATTGTGGAAGG & GGATGCAGGGATGATGTTCT \\
HMGCR & GACGGTGACACTTACCATCTGT & GATGCACCGTGTTATGGTGA \\
HNF1 $\alpha$ & GAGGTGGCTCAGCAATTCAC & CACTCCTCCACCAAGGTCTC \\
HNF $1 \beta$ & CAGCCAGTCGGTTTTACAGC & ATTCGTCAAGGTGCTGACGG \\
LDLR & TTGGGTTGATTCCAAACTCC & GATTGGCACTGAAAATGGCT \\
PCSK9 & TGCTCCAGAGGTCATCACAG & GTCCCACTCTGTGACATGAAG \\
SREBP1 & GCACTTTTTGACACGTTTCTTC & CTGTACAGGCTCTCCTGTGG
\end{tabular}

HNF, hepatocyte nuclear factor; SREBP, sterol regulatory element-binding protein; PCSK9, proprotein convertase subtilisin/kexin type 9; HMGCR, HMG- CoA reductase; LDLR, LDL receptor.

then sequentially incubated with PCSK9 conjugate followed by the PCSK9 substrate solution with extensive intermittent washes between each step. The amount of PCSK9 in serum was estimated colorimetrically using a standard microplate reader (MDS Analytical Technologies, Silicon Valley, CA, USA). Additionally, PCSK9 in hamster serum samples was detected by immunoprecipitation, followed by western blot analysis using anti-hamster PCSK9 antibody as we described previously $(21,22)$.

$R N A$ isolation and reverse transcription-quantitative $P C R(R T-q P C R)$. Total RNA was isolated from $20 \mathrm{mg}$ of individual flash frozen mouse liver tissue samples using the RNeasy PLUS Mini kit (Qiagen, Inc., Valencia, CA, USA). After RNA integrity was confirmed by gel electrophoresis, $1.5 \mu \mathrm{g}$ of total RNA was reverse transcribed by random priming using the High-Capacity cDNA reverse transcription kit (Invitrogen Life Technologies) according to the manufacturer's guidelines. Quantitative PCR (qPCR) was then performed in an ABI 7900HT sequence detection system using SYBR-Green PCR Master Mix (Invitrogen Life Technologies) and PCR primers specific for each gene being amplified (Table I). With duplicate measurements from each cDNA sample, the data were analyzed using the $\Delta \Delta C T$ method and the relative expression of target mRNA level was normalized to that of GAPDH in each sample.

Western blot analysis. Total protein was extracted from flash-frozen mouse liver samples by homogenizing $50 \mathrm{mg}$ of tissue in RIPA buffer supplemented with $1 \mathrm{mM} \mathrm{PMSF}$ and protease inhibitor cocktail (Roche, Mannheim, Germany). Protein content was quantified using BCA protein assay reagent (Pierce) and $50 \mu \mathrm{g}$ of protein from individual samples was resolved by SDS-PAGE. Following transfer onto nitrocellulose membranes, LDLR, PCSK9, HNF $1 \alpha$ and $\beta$-actin proteins were detected by immunoblotting using a rabbit anti-LDLR (BioVision, Inc., Milpitas, CA, USA), anti-PCSK9 (8), goat anti-HNF1 $\alpha$ (sc-6547; Santa Cruz Biotechnology, Inc., Dallas, TX, USA) and monoclonal anti- $\beta$-actin (Clone AC-15; Sigma-Aldrich, St. Louis, MO, USA) antibodies. Immunoreactive bands of predicted molecular mass were visualized using SuperSignal West Femto chemiluminescent substrate (Pierce Chemical Co., Rockford, IL, USA) and
FluorChem E western blot analysis imaging system (Protein Simple, San Jose, CA, USA). Background subtracted band densities were quantified using Alpha View SA imaging software (Protein Simple). Signal intensities for $\beta$-actin were used to normalize differences in protein loading among individual samples.

Statistical analysis. All values are expressed as mean \pm SEM. One way ANOVA with Dunnett's post-test was performed using Prism 5 software (GraphPad Software Inc., La Jolla, CA, USA). Statistical significance is displayed as $\mathrm{P}<0.05, \mathrm{P}<0.01$ or $\mathrm{P}<0.001$.

\section{Results}

Cloning of hamster HNFI $\alpha$ coding region and adenovirus-mediated gene knockdown. To assess the role of HNF1 $\alpha$ in hepatic PCSK9 transcription in hamster species, we first cloned the entire coding region of hamster HNF1 $\alpha$ orthologue from cDNAs generated from hamster liver tissue. Hamster HNF1 $\alpha$ protein sequence is highly homologous to human, mouse and rat species with amino acid sequence identity in the range of $94-97 \%$. Based upon the cDNA sequence, we constructed several plasmids that express shRNAs targeting different coding regions of hamster HNF1 $\alpha$. One of the shRNAs targets hamster HNF1 $\alpha$ mRNA coding sequence from 786-806 that is identical to human, mouse and rat sequences (Fig. 1A).

First, to demonstrate an effective knockdown of HNF1 $\alpha$ by this shRNA in hamsters without RSV treatment, we infected two hamsters with Ad-shHNF1 $\alpha$ and another two hamsters with control virus Ad-shLacZ for 10 days. Hepatic gene expression analysis by qPCR demonstrated that the mRNA levels of HNF1 $\alpha$ and PCSK9 were both reduced by $~ 40-50 \%$ in hamsters injected with Ad-shHNF1 $\alpha$ as compared to hamsters injected with Ad-shLacZ. By contrast, the mRNA expression of LDLR was not affected by HNF1 $\alpha$ knockdown (Fig. 1B). Reductions of HNF1 $\alpha$ and PCSK9 mRNA expression by the shRNA expression were further corroborated by western blot analysis that showed reduced liver HNF1 $\alpha$ and PCSK9 protein levels and increased LDLR protein abundance in both hamsters infected with Ad-shHNF1 $\alpha$ (Fig. 1C). Taken 


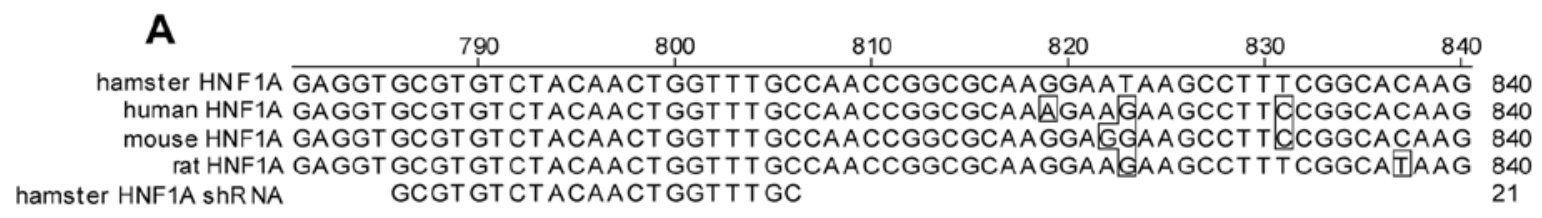

B

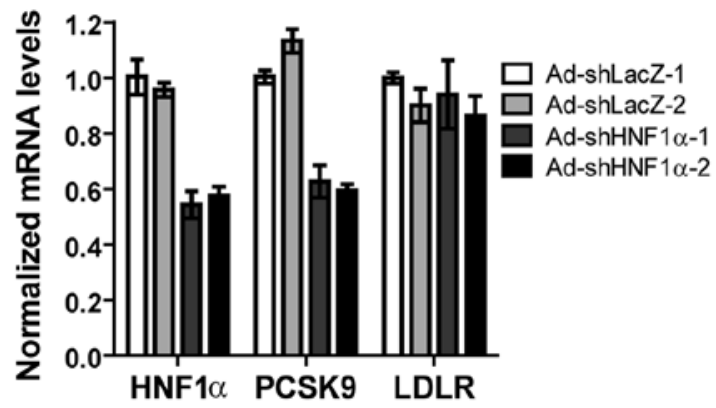

C

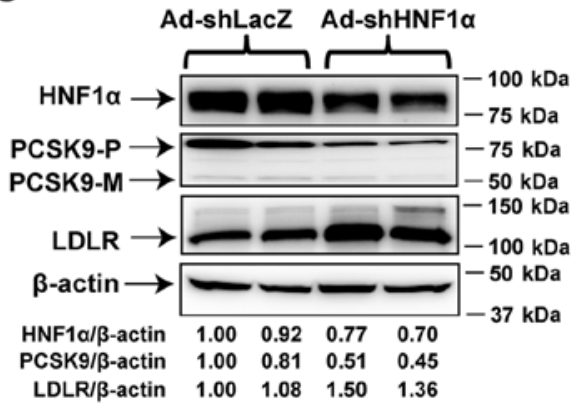

\begin{abstract}
D
1450

1460

1470

1480

1490

1500

Ham HNF1B X1 AGGAGGAGGACT GCCCCCCGT CAGCACCT TGACGAATATCCACAGCCTCTCCCACCACAA 1280 Ham HNF1B $\times 2$ AGGAGGAGGACT GCCCCCCGT CAGCACCTTGACGAATATCCACAGCCTCTCCCACCACAA 1497 Ham HNF1B X3 AGGAGGAGGACT GCCCCCCGT CAGCACCTTGACGAATATCCACAGCCTCTCCCACCACAA 1202 Ham HNF1B X4 AGGAGGAGGACT GCCCCCCGTCAGCACCTTGACGAATATCCACAGCCTCTCCCACCACAA 1419 Ham HNF1B X5 AGGAGGAGGACTGCCCCCCGTCAGCACCTTGACGAATATCCACAGCCTCTCCCACCACAA 1332 human HNF1B AGGAGGAGGTTT GCCCCCAGT CAGCACCTTGACGAATATCCACAGCCTCTCCCACCATAA 1318 mouse HNF1B Sh HNF1B GCACCTTGACGAATATCCACA

Figure 1. shRNA sequence alignment and validation of shHNF1 $\alpha$-targeted knockdown in hamsters. (A) The sequence of HNF1 $\alpha$ shRNA is aligned with its target sequences in the HNF1 $\alpha$-coding sequences of hamster, human, mouse and rat species. Nucleotides of human, mouse and rat HNF1 $\alpha$ that differ from those of the hamster sequence are boxed. (B) Two male Syrian golden hamsters were injected with Ad-shLacZ or Ad-shHNF1 $\alpha$ (5x10 ${ }^{11}$ virus particles per hamster) adenoviral particles. Ten days after the viral injection, all animals were euthanized and liver total RNA was isolated. Individual levels of HNF1o mRNA, LDLR mRNA and PCSK9 mRNA were assessed by quantitative real-time PCR using hamster-specific PCR primers as described in the Materials and methods section. After normalization with GAPDH mRNA levels, the relative expression levels of mRNAs of interest are presented. The results are expressed as the means \pm SEM of triplicate measurements of each cDNA sample of individual hamsters. (C) Individual liver protein extracts were prepared and protein concentrations were determined. A total of $50 \mu \mathrm{g}$ of homogenate proteins from individual liver samples was analyzed by western blot analysis and the expression levels of LDLR, PCSK9 and HNF1 $\alpha$ were quantified by Alpha View software with normalization to signals of $\beta$-actin. (D) The sequence of HNF1 $\beta$ shRNA is aligned with its target sequences in the HNF1 $\beta$-coding sequences of 5 predicted isoforms of hamster, human and mouse HNF1 $\beta$. Nucleotides of human and mouse HNF1 $\beta$ that differ from those of the hamster sequence are boxed. HNF1, hepatocyte nuclear factor 1; PCSK9, proprotein convertase subtilisin/kexin type 9 .

together, these results validated the success of this shRNA in knockdown of HNF1 $\alpha$ expression and demonstrated its impact on PCSK9 expression in hamsters without statin treatment.

To examine the potential role of HNF1 $\beta$ in PCSK9 transcription in hamster species, from a hamster liver cDNA pool, we cloned a partial coding region of hamster HNF1 $\beta$ and sequenced. The sequence of the partial clone was identical to the corresponding sequence of the NCBI-predicted HNF1 $\beta$ transcript X1. A sequence spanning nucleotides 1464-1484 of the hamster HNF1 $\beta$ transcript X1 was targeted for shRNAmediated knockdown. Of note, this shRNA is also capable of targeting other predicted hamster HNF1 $\beta$ transcripts as well as mouse and human transcripts (Fig. 1D). The specificity of this HNF1 $\beta$ shRNA has been validated in cultured human and mouse hepatic cells and in mouse liver tissue (17).

Knockdown of HNFl $\alpha$ or HNF1 $\beta$ in hamsters abolishes the RSV-induced elevation of serum PCSK9 levels. Since our previous study showed that RSV treatment significantly increased circulating PCSK9 levels in dyslipidemic hamsters (12), we wanted to know whether knockdown of HNF1 $\alpha$ or HNF1 $\beta$ in hamsters could antagonize the
RSV-induced elevation of serum PCSK9 levels. Hamsters were injected with Ad-shHNF1 $\alpha$, Ad-shHNF1 $\beta$ or a control virus (Ad-shLacZ). Three days after viral infection, the animals were orally administered RSV (15 mg/kg/day) or vehicle for 7 days. The results of RT-qPCR confirmed the successful knockdown of both HNF1 $\alpha$ and HNF1 $\beta$ by the hepatic expression of their specific shRNAs (Fig. 2A). The mRNA level of HNF1 $\alpha$ was not changed by RSV treatment, but it was reduced to $60 \%$ of the control by injection of Ad-shHNF1 $\alpha$ into hamsters. Likewise, the expression levels of HNF1 $\beta$ mRNA were unaffected by the RSV treatment but they were reduced to $60 \%$ of the control by Ad-shHNF1 $\beta$ injection and to $70 \%$ of the control by Ad-shHNF1 $\alpha$ injection. The inhibition of HNF $1 \beta$ mRNA expression by Ad-shHNF1 $\alpha$ was also observed in our previous study in mice (17). RSV treatment significantly increased the PCSK9 mRNA level by $94 \%(\mathrm{P}<0.01)$ in the control hamsters but not in hamsters injected with Ad-shHNF1 $\alpha$ or Ad-shHNF1 $\beta$ (Fig. 2A). Furthermore, RSV treatment elevated serum PCSK9 levels by $\sim 32.5 \%(\mathrm{P}<0.05)$ in the wild-type hamsters (Ad-shLacZ). Expression of HNF1 $\alpha$ shRNA or HNF1 $\beta$ shRNA in the liver of hamsters both prevented the RSV-induced elevation of PCSK9 serum levels (Fig. 2B). 
A
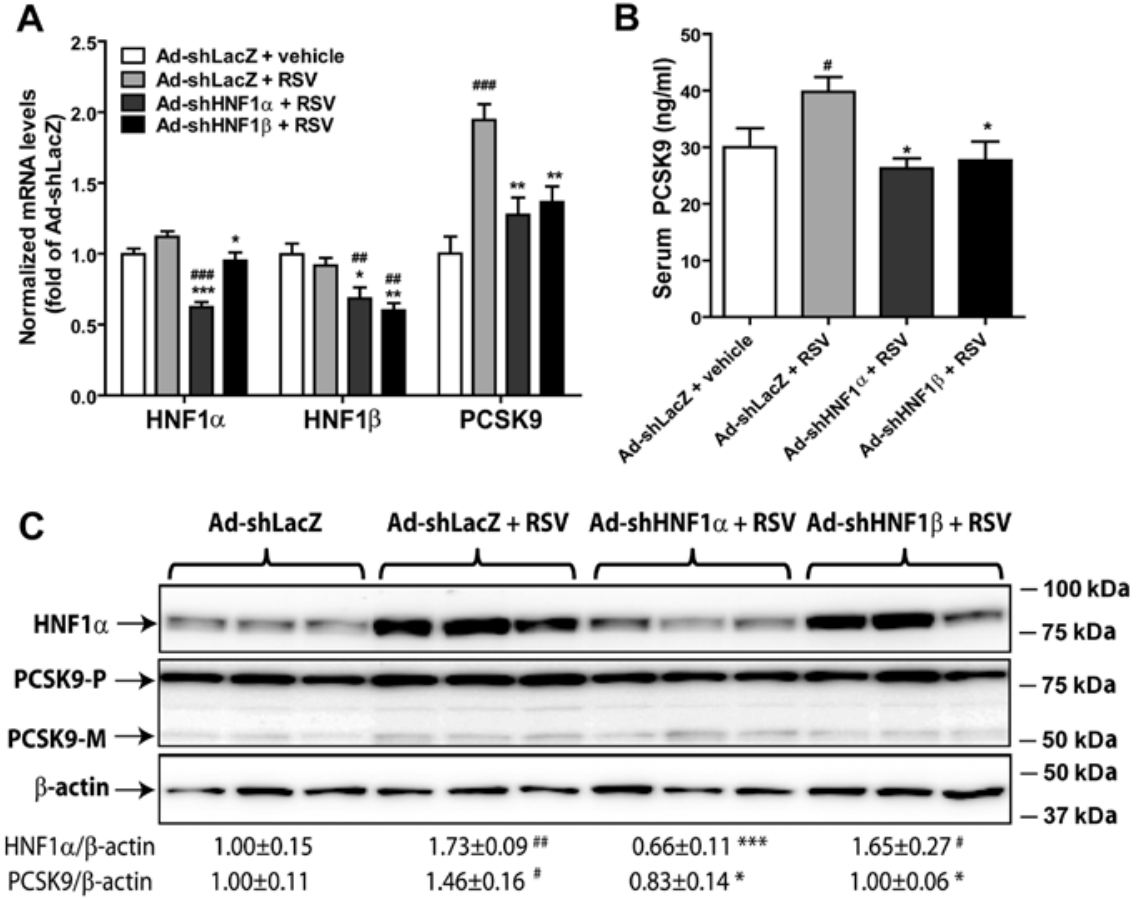

Figure 2. Knockdown of HNF1 $\alpha$ or HNF1 $\beta$ in the hamster reduces serum PCSK9 levels in hamsters treated with RSV. Hamsters fed a normal diet were injected with $5 \times 10^{11}$ virus particles/hamster of Ad-shLacZ, Ad-shHNF1 $\alpha$ or Ad-shHNF1 $\beta$ adenovirus particles (n=6 animals/treatment). At day 3 of injection, RSV at a daily dose of $15 \mathrm{mg} / \mathrm{kg}$ was orally administered to hamsters for 7 days. Four-hour fasted serum samples were collected on day 10 post infection. (A) Real-time PCR analysis of hamster liver mRNA levels of HNF1 $\alpha$, HNF1 $\beta$ and PCSK9. (B) Individual hamster serum PCSK9 levels were quantified by a mouse PCSK9 ELISA kit. Values are expressed as the mean \pm SEM. (C) Western blot analysis of hamster liver protein levels of HNF1 $\alpha$ and PCSK9. Three representative liver samples/group are shown. The protein abundances of PCSK 9 and HNF1 $\alpha$ were quantified using Alpha View software. Values are expressed as the mean \pm SEM of 5 randomly chosen liver samples/group. ${ }^{\#} \mathrm{P}<0.05,{ }^{\# \#} \mathrm{P}<0.01$ and ${ }^{\# \# \#} \mathrm{P}<0.001$ as compared to the Ad-shLacZ group. ${ }^{*} \mathrm{P}<0.05$ and ${ }^{* * *} \mathrm{P}<0.001$ as compared to the Ad-shLacZ + RSV group. HNF1, hepatocyte nuclear factor 1; RSV, rosuvastatin; PCSK9, proprotein convertase subtilisin/kexin type 9.

Examination of HNF1 $\alpha$ protein content by western blot analysis showed that the liver HNF1 $\alpha$ protein level was significantly induced by RSV treatment (Fig. 2C), that confirmed our previous finding made in dyslipidemic hamsters (12). Injection of Ad-shHNF1 $\alpha$, but not Ad-shHNF1 $\beta$, significantly reduced liver HNF1 $\alpha$ protein levels, further demonstrating the specificity of the shRNA. Because an antibody against hamster HNF1 $\beta$ is not available, we were unable to measure liver HNF1 $\beta$ protein levels in this study. Importantly, western blot analysis showed that RSV treatment increased the hepatic PCSK9 protein amount. This effect was again abolished by adenovirus-directed expressions of HNF1 shRNAs (Fig. 2C).

Depletion of HNF1 isoforms increases liver LDLR protein content and reduces serum cholesterol levels. Our previous study on dyslipidemic hamsters detected a reduced hepatic LDLR content in RSV-treated hamsters (12). This finding was observed again in the normolipidemic hamsters treated with RSV in the wild-type animals (Ad-shLacZ) (Fig. 3A). However, knockdown of HNF1 $\alpha$ as well as HNF1 $\beta$ both prevented the negative effect of RSV and further increased LDLR protein levels to $135 \%(\mathrm{P}<0.001)$ and $142 \%$ of the control $(\mathrm{P}<0.001)$, respectively.

In these normolipidemic hamsters, RSV treatment showed a trend in the elevation of serum TC and non-HDL cholesterol levels in the control hamsters (Fig. 3B and 3C). However, injection of Ad-shHNF1 $\alpha$ or Ad-shHNF1 $\beta$ into hamsters under RSV treatment reduced serum cholesterol levels. Serum TC and non-high density lipoprotein cholesterol (HDL-C) levels were $18 \%(\mathrm{P}<0.05)$ and $27 \%(\mathrm{P}<0.05)$ lower in the Ad-shHNF1 $\alpha+\mathrm{RSV}$ group as compared with the Ad-shLacZ + RSV group, respectively. Likewise, serum TC was reduced by $18 \%(\mathrm{P}<0.05)$ and non-HDL-C was lowered by $32 \%(\mathrm{P}<0.05)$ in the Ad-shHNF1 $\beta+\mathrm{RSV}$ group compared to the control. Liver cholesterol levels were similar among all groups. Furthermore, serum levels of liver transaminase ALT were not elevated by adenoviral injection or RSV treatment (data not shown), suggesting that the normal function of the liver was not disrupted in these animals.

Hepatic depletion of HNF1 isoforms does not affect the SREBP pathway. The transcription of PCSK9 is controlled by both SRE-1 and HNF1 sites on its promoter region. To examine the involvement of SRE-1, we measured mRNA levels of LDLR and HMG-CoA reductase (HMGCR) in all groups. Fig. 4A showed that RSV treatment increased the mRNA levels of LDLR and HMGCR. However, in contrast to PCSK9, injection of Ad-shHNF1 $\alpha$ or Ad-shHNF1 $\beta$ did not inhibit RSV-induced mRNA expression of these SRE target genes. We further examined the liver contents of mature SREBR2 in different liver samples by western blot analysis and demonstrated that the induction of mSREBP2 by RSV treatment was unaffected by the knockdown of either HNF1 $\alpha$ or HNF1 $\beta$ (Fig. 4B). This data excluded the involvement of the SREBP2 pathway in the reduction of PCSK9 transcription in liver tissues with specific knockdown of HNF1 $\alpha$ and HNF1 $\beta$. 

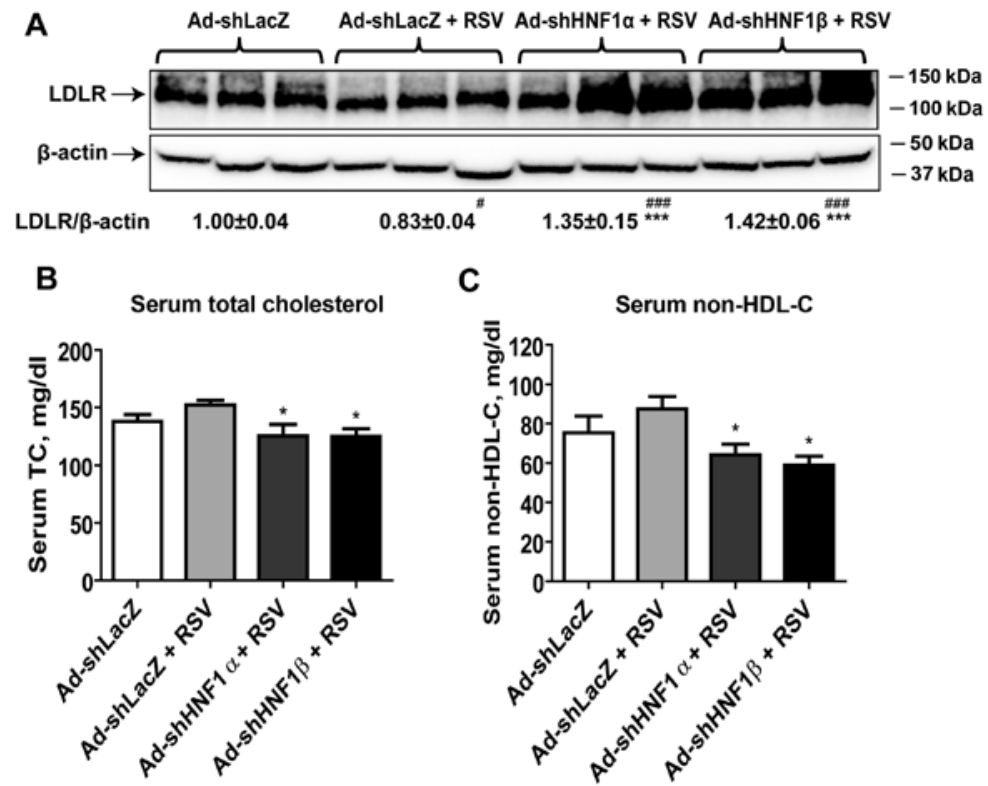

Figure 3. Hepatic depletion of HNF1 isoforms increases liver LDLR protein contents and reduces serum cholesterol levels. Hamsters fed a normal diet were injected with Ad-shLacZ, Ad-shHNF1 $\alpha$ or Ad-shHNF1 $\beta$ ( $\mathrm{n}=6$ animals/treatment). At day 3 of injection, RSV at a daily dose of $15 \mathrm{mg} / \mathrm{kg}$ was orally administered to hamsters for 7 days. Four-hour fasted serum samples were collected on day 10 post infection. (A) Western blot analysis of hamster liver protein levels of LDLR. Three representative liver samples are shown and the quantitation was performed from analysis of 5 liver samples per group as described in Fig. 1C. (B) Individual hamster serum total cholesterol levels were measured. (C) Individual hamster serum HDL-C levels were measured. The concentrations of non-HDL-C were derived after subtraction of HDL-C from total cholesterol. " $\mathrm{P}<0.05$ as compared to the Ad-shLacZ + RSV group. LDLR, LDL receptor; HNF1, hepatocyte nuclear factor 1 .

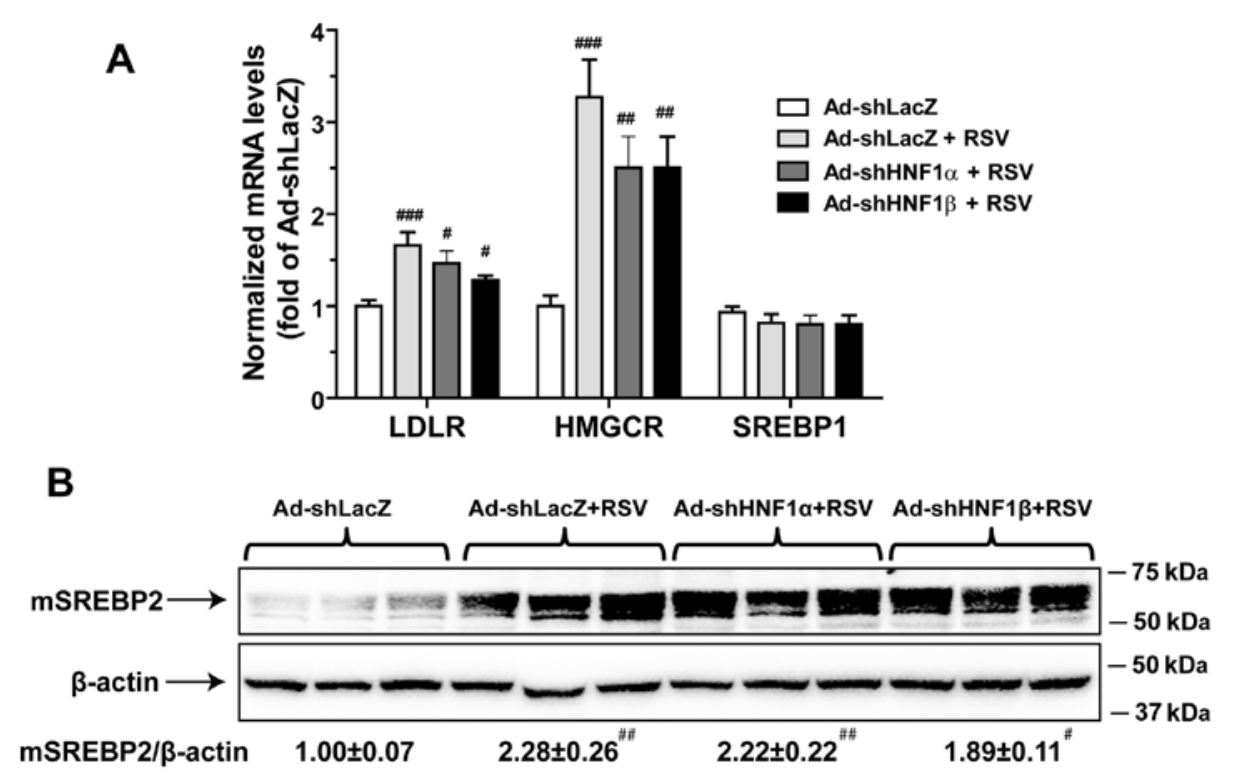

Figure 4. Depletion of HNF1 factors does not affect RSV-induced activation of the SREBP pathway. Real-time PCR analysis of hamster liver mRNA levels of LDLR and HMGCR was performed. (A) Values are expressed as the mean \pm SEM of 6 samples/group. ${ }^{\#} \mathrm{P}<0.05$, ${ }^{\# \#} \mathrm{P}<0.01$ and ${ }^{\# \# \#} \mathrm{P}<0.001$ as compared to the Ad-shLacZ group. (B) Fifty micrograms of homogenate proteins of 3 randomly chosen liver samples of the same treatment group was resolved by SDS-PAGE. mSREBP2 was detected by immunoblotting using the anti-SREBP2 antibody. The membrane was reprobed with an anti- $\beta$-actin antibody. The protein abundance of mSREBP2 was quantified using Alpha View software. Values are expressed as the mean \pm SEM of five randomly chosen liver samples per group. ${ }^{\#} \mathrm{P}<0.05$ and ${ }^{\# \#} \mathrm{P}<0.01$ as compared to the Ad-shLacZ + RSV group. HNF1, hepatocyte nuclear factor 1; RSV, rosuvastatin; HMGCR, HMG-CoA reductase; SREBP, sterol regulatory element-binding protein

\section{Discussion}

PCSK9 has become an important new therapeutic target for the management of hyperlipidemia and reduction in cardiovascular disease. With the rapid development of anti-PCSK9 antibody as new therapy (23-25), many patients with high circulating LDL-C levels could be treated with PCSK9 inhibitors to drastically reduce serum PCSK9 levels to protect liver LDLR from degradation. On the other hand, our current understanding of the physiological regulation of PCSK9 expression at the transcriptional level as well as the translational level remains incomplete. 
In the present study, we applied the approach of adenovirus-mediated gene knockdown to investigate the individual roles of HNF1 $\alpha$ and HNF1 $\beta$ in circulating PCSK9 levels in the hamster, a species that shares more characteristics in lipid metabolism with humans than any other rodents including mice and rats (26-30). This study led to several important new findings.

First, we demonstrated that liver-specific depletion of HNF1 $\alpha$ in hamsters without statin treatment reduced PCSK9 mRNA levels by $40 \%$ and increased hepatic LDLR levels substantially. These results are highly consistent with the effect of HNF1 $\alpha$ knocking down in normolipidemic mice, and further underscore the physiological role of HNF1 $\alpha$ as a transactivator for PCSK9 gene expression in liver tissue of different animal models.

Secondly, our previous study in dyslipidemic hamsters showed that in addition to an increase in SREBP2 expression, RSV treatment increased the liver abundance of HNF1 $\alpha$ protein (12). Our study suggested that the inducing effect of RSV on HNF1 $\alpha$ is likely an underlying mechanism accounting for the higher induction of PCSK9 than LDLR due to the utilization of two transactivators (HNF1 $\alpha$ and SREBP2) in PCSK9 transcription versus one (SREBP2) in LDLR transcription. From this perspective, HNF1 $\alpha$ shRNA could act as an antagonist to directly inhibit RSV-induced PCSK9 expression. Thus, in this investigation, we focused on the examination of the effects of hepatic depletion of HNF1 $\alpha$ in hamsters under RSV treatment. Indeed, injection of Ad-shHNF1 $\alpha$ into hamsters totally abolished RSV-induced elevation in serum PCSK9 and hepatic PCSK9 mRNA and protein levels. This led to a marked increase in liver LDLR and significant reductions in serum cholesterol levels. Interestingly, we again observed the elevated HNF1 $\alpha$ protein levels in the liver of RSV-treated hamsters. The precise mechanism by which RSV affects HNF1 $\alpha$ protein abundance is presently unclear.

The third important outcome of our study is the demonstration of the nonexpendable role of HNF1 $\beta$ in PCSK9 transcription in hamster liver tissue. We demonstrated that injection of Ad-shHNF1 $\beta$ into hamsters substantially lowered serum PCSK9 and hepatic PCSK9 to levels nearly identical to the effects generated by depleting HNF1 $\alpha$. The fact that expression levels of HNF1 $\alpha$ protein and mRNA were not reduced in hamster livers with HNF1 $\beta$ knockdown excluded the possibility of the cross-reactivity of HNF1 $\beta$ shRNA. The strong inhibitory effects of HNF1 $\beta$ shRNA on PCSK9 expression in the hamsters were strikingly different from the results following the knockdown of HNF1 $\beta$ in mouse liver. In our previous study conducted in mice, we did not observe any inhibition of PCSK9 expression levels in both liver and serum by Ad-shHNF1 $\beta$-directed expression of HNF1 $\beta$ shRNA. The lack of effect on PCSK9 expression was not due to insufficient depletion of HNF1 $\beta$ because we showed that the HNF1 $\beta$ mRNA abundance and the mRNA expression of three HNF1 $\beta$ target genes (ApoC3, HNF4 $\alpha$ and FABP5) were all greatly reduced in the liver of the Ad-shHNF1 $\beta$-infected mice.

The different roles of HNF1 $\beta$ in PCSK9 transcription between the hamster and mouse could be caused by variations in species-specific expression of mature transcripts. By quantitative PCR measurement, it was reported that in the mouse adult liver, HNF1 $\alpha$ is a dominant isoform with mRNA levels more than 7-fold the levels of HNF1 $\beta$ (31). We compared the relative mRNA levels of HNF1 $\alpha$ and HNF1 $\beta$ in hamster liver and showed that HNF1 $\beta$ mRNA was expressed at a level of $31 \%$ of that of HNF1 $\alpha$ mRNA, which is substantially higher than the HNF1 $\beta$ mRNA amount detected in mouse liver. We attempted to detect HNF1 $\beta$ protein levels in liver tissue using several commercial anti-HNF1 $\beta$ antibodies. However, none of these produced specific signals on western blot analyses. Thus, it is unclear whether RSV treatment also increased HNF1 $\beta$ protein levels. Another possible explanation for the effectiveness of HNF1 $\beta$ shRNA on RSV-induced PCSK9 expression is that in hamster liver, HNF1 $\alpha$ and HNF1 $\beta$ might bind to the HNF1 site of the PCSK9 promoter as an obligated heterodimer. Thus, reducing either one would attenuate the HNF1-mediated transactivation of the PCSK9 gene.

In conclusion, we demonstrated that liver-specific knockdown of HNF1 $\alpha$ or HNF1 $\beta$ in hamsters produced antagonistic effects on the RSV-induced elevation of serum PCSK9 and cholesterol levels. Thus, both HNF1 $\alpha$ and HNF1 $\beta$ play key roles in the control of PCSK9 gene transcription in liver tissue of the hamster species. Combined with the difference observed in Ad-shHNF1 $\beta$-mediated knockdown between hamsters and mice and the strong induction of HNF1 $\alpha$ protein by RSV treatment in hamster liver tissue, our study results further support the notion that the regulatory mechanism governing PCSK9 expression is species-specific, which could be a contributing factor for different efficacies of statins and other lipid-modulating drugs observed in various animal studies. The question of whether statins regulate hepatic HNF1 $\alpha$ in other species including humans has clinical relevance and requires further investigation.

\section{Acknowledgements}

The present study was supported by the Department of Veterans Affairs (Office of Research and Development, Medical Research Service) and by grants (no. 1R01AT006336-01A1) from the National Center of Complementary and Alternative Medicine.

\section{References}

1. Zhang DW, Lagace TA, Garuti R, Zhao Z, McDonald M, Horton JD, Cohen JC and Hobbs HH: Binding of proprotein convertase subtilisin/kexin type 9 to epidermal growth factor-like repeat A of low density lipoprotein receptor decreases receptor recycling and increases degradation. J Biol Chem 282: 18602-18612, 2007.

2. Lagace TA, Curtis DE, Garuti R, McNutt MC, Park SW, Prather HB, Anderson NN, Ho YK, Hammer RE and Horton JD: Secreted PCSK9 decreases the number of LDL receptors in hepatocytes and in livers of parabiotic mice. J Clin Invest 116: 2995-3005, 2006.

3. Lambert G, Ancellin N, Charlton F, Comas D, Pilot J, Keech A, Patel S, Sullivan DR, Cohn JS, Rye KA and Barter PJ: Plasma PCSK9 concentrations correlate with LDL and total cholesterol in diabetic patients and are decreased by fenofibrate treatment. Clin Chem 54: 1038-1045, 2008.

4. Lakoski SG, Lagace TA, Cohen JC, Horton JD and Hobbs HH: Genetic and metabolic determinants of plasma PCSK9 levels. J Clin Endocrinol Metab 94: 2537-2543, 2009.

5. Ling H, Burns TL and Hilleman DE: An update on the clinical development of proprotein convertase subtilisin kexin 9 inhibitors, novel therapeutic agents for lowering low-density lipoprotein cholesterol. Cardiovasc Ther 32: 82-88, 2014. 
6. Horton JD, Shah NA, Warrington JA, Anderson NN, Park SW, Brown MS and Goldstein JL: Combined analysis of oligonucleotide microarray data from transgenic and knockout mice identifies direct SREBP target genes. Proc Natl Acad Sci USA 100: 12027-12032, 2003.

7. Maxwell KN, Soccio RE, Duncan EM, Sehayek E and Breslow JL: Novel putative SREBP and LXR target genes identified by microarray analysis in liver of cholesterol-fed mice. J ipid Res 44: 2109-2119, 2003.

8. Li H, Dong B, Park SW, Lee HS, Chen W and Liu J: Hepatocyte nuclear factor 1alpha plays a critical role in PCSK9 gene transcription and regulation by the natural hypocholesterolemic compound berberine. J Biol Chem 284: 28885-28895, 2009.

9. Dubuc G, Chamberland A, Wassef H, Davignon J, Seidah NG, Bernier L and Prat A: Statins upregulate PCSK9, the gene encoding the proprotein convertase neural apoptosis-regulated convertase-1 implicated in familial hypercholesterolemia. Arterioscler Thromb Vasc Biol 24: 1454-1459, 2004.

10. Jeong HJ, Lee HS, Kim KS, Kim YK, Yoon D and Park SW: Sterol-dependent regulation of proprotein convertase subtilisin/kexin type 9 expression by sterol-regulatory element binding protein-2. J Lipid Res 49: 399-409, 2008.

11. Raal F, Panz V, Immelman A and Pilcher G: Elevated PCSK9 levels in untreated patients with heterozygous or homozygous familial hypercholesterolemia and the response to high-dose statin therapy. J Am Heart Assoc 2: e000028, 2013.

12. Dong B, Wu M, Li H, Kraemer FB, Adeli K, Seidah NG, Park SW and Liu J: Strong induction of PCSK9 gene expression through HNF1alpha and SREBP2: Mechanism for the resistance to LDL-cholesterol lowering effect of statins in dyslipidemic hamsters. J Lipid Res 51: 1486-1495, 2010.

13. Costa RH, Kalinichenko VV, Holterman AX and Wang X: Transcription factors in liver development, differentiation, and regeneration. Hepatology 38: 1331-1347, 2003.

14. Mendel DB, Hansen LP, Graves MK, Conley PB and Crabtree R: HNF-1 $\alpha$ and HNF-1 $\beta$ (vHNF-1) share dimerization and homeo domains, but not activation domains, and form heterodimers in vitro. Genes Dev 5: 1042-1056, 1991.

15. Ai D, Chen C, Han S, Ganda A, Murphy AJ, Haeusler R, Thorp E, Accili D, Horton JD and Tall AR: Regulation of hepatic LDL receptors by mTORC1 and PCSK9 in mice. J Clin Invest 122 : 1262-1270, 2012.

16. Servitja J-M, Pignatelli M, Maestro MA, Cardalda C, Boj SF, Lozano J, Blanco E, Lafuente A, McCarthy MI, Sumoy L, et al: Hnfla (MODY3) controls tissue-specific transcriptional programs and exerts opposed effects on cell growth in pancreatic islets and liver. Mol Cell Biol 29: 2945-2959, 2009.

17. Shende VR, Wu M, Singh AB, Dong B, Kan CF and Liu J: Reduction of circulating PCSK9 and LDL-C levels by liver-specific knockdown of HNF1 $\alpha$ in normolipidemic mice. J Lipid Res 56: 801-809, 2015.

18. Dong B, Singh AB, Azhar S, Seidah NG and Liu J: High-fructose feeding promotes accelerated degradation of hepatic LDL receptor and hypercholesterolemia in hamsters via elevated circulating PCSK9 levels. Atherosclerosis 239: 364-374, 2015.
19. Miao J, Manthena PV, Haas ME, Ling AV, Shin DJ, Graham MJ, Crooke RM, Liu J and Biddinger SB: Role of insulin in the regulation of proprotein convertase subtilisin/kexin type 9. Arterioscler Thromb Vasc Biol 35: 1589-1596, 2015.

20. Hentze H, Jensen KK, Chia SM, Johns DG, Shaw RJ, Davis HR Jr, Shih SJ and Wong KK: Inverse relationship between LDL cholesterol and PCSK9 plasma levels in dyslipidemic cynomolgus monkeys: Effects of LDL lowering by ezetimibe in the absence of statins. Atherosclerosis 231: 84-90, 2013.

21. Dong B, Li H, Singh AB, Cao A and Liu J: Inhibition of PCSK9 transcription by berberine involves down-regulation of hepatic HNF1 $\alpha$ protein expression through the ubiquitin-proteasome degradation pathway. J Biol Chem 290: 4047-4058, 2015.

22. Cao A, Wu M, Li H and Liu J: Janus kinase activation by cytokine oncostatin M decreases PCSK9 expression in liver cells. J Lipid Res 52: 518-530, 2011.

23. Wu M, Dong B, Cao A, Li $\mathrm{H}$ and Liu J: Delineation of molecular pathways that regulate hepatic PCSK9 and LDL receptor expression during fasting in normolipidemic hamsters. Atherosclerosis 224: 401-410, 2012.

24. Verbeek R, Stoekenbroek RM and Hovingh GK: PCSK9 inhibitors: Novel therapeutic agents for the treatment of hypercholesterolemia. Eur. J Pharmacol 763-: 38-47, 2015.

25. Huynh K: Dyslipidaemia. Assessing the efficacy and safety of evolocumab and alirocumab. Nat Rev Cardiol 12: 261,2015.

26. Desai NR and Sabatine MS: PCSK9 inhibition in patients with hypercholesterolemia. Trends Cardiovasc Med 25: 567-574, 2015.

27. Yin W, Carballo-Jane E, McLaren DG, Mendoza VH, Gagen K, Geoghagen NS, McNamara LA, Gorski JN, Eiermann GJ, Petrov A, et al: Plasma lipid profiling across species for the identification of optimal animal models of human dyslipidemia. J Lipid Res 53: 51-65, 2012.

28. Dietschy JM, Turley SD and Spady DK: Role of liver in the maintenance of cholesterol and low density lipoprotein homeostasis in different animal species, including humans. J Lipid Res 34: 1637-1659, 1993.

29. Quig DW, Arbeeny CM and Zilversmit DB: Effects of hyperlipidemias in hamsters on lipid transfer protein activity and unidirectional cholesteryl ester transfer in plasma. Biochim Biophys Acta 1083: 257-264, 1991

30. Taghibiglou C, Carpentier A, Van Iderstine SC, Chen B, Rudy D, Aiton A, Lewis GF and Adeli K: Mechanisms of hepatic very low density lipoprotein overproduction in insulin resistance. Evidence for enhanced lipoprotein assembly, reduced intracellular ApoB degradation, and increased microsomal triglyceride transfer protein in a fructose-fed hamster model. J Biol Chem 275: 8416-8425, 2000.

31. Harries LW, Brown JE and Gloyn AL: Species-specific differences in the expression of the HNF1A, HNF1B and HNF4A genes. PLoS One 4: e7855, 2009. 